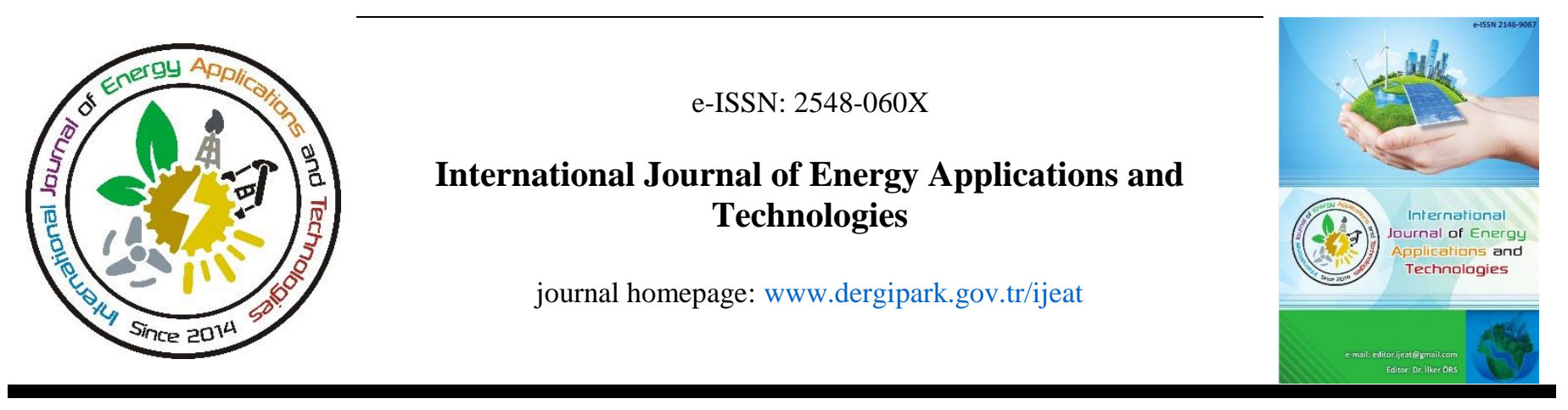

Original Research Article

\title{
Experimental flow analysis of vertical axis turbine for power generation in open channel system
}

\author{
Bülent Yanıktepe*, Ertuğul Şekeroğlu, Mustafa Söyler, Coşkun Özalp \\ Department of Energy Systems Engineering, Faculty of Engineering, Osmaniye Korkut Ata University, Osmaniye, Turkey
}

\author{
ARTICLE INFO \\ * Corresponding author \\ byaniktepe@osmaniye.edu.tr \\ Received October 7, 2020 \\ Accepted November 23, 2020 \\ Published by Editorial Board \\ Members of IJEAT \\ (C) This article is distributed by \\ Turk Journal Park System under \\ the CC 4.0 terms and conditions. \\ doi: 10.31593/ijeat.806950
}

\begin{abstract}
In this experimental study, flow structure over a three bladed vertical axis Darrieus turbine is investigated experimentally for Reynold Number, $\mathrm{Re}=9000$ in an open channel. Rigidity ratio $(\sigma)$, angle of attack, optimum values of blade chord length $\left(C_{L}\right)$, turbine radius $(R)$ and height of turbine blades $(\mathrm{H})$ are used as approximately 1.1 and $\alpha=-2^{\circ}, \mathrm{C}_{\mathrm{L}}=5 \mathrm{~cm}, \mathrm{R}=13.75 \mathrm{~cm}$ and $\mathrm{H}=20$ $\mathrm{cm}$, respectively. According to the values, $1 / 4$ scale model of a vertical axis Darrieus turbine has been used in order to perform experimental flow analysis. In experimental flow analysis, two different azimuth angles, $\theta=270^{\circ}$ and $\theta=240^{\circ}$ are used to examine the flow characteristics in the Particle İmage Velocimetry (PIV) experiments. As a result of the experiments, time averaged velocity vectors $\langle\mathrm{V}\rangle$, averaged streamlines $\langle\Psi\rangle$, and averaged vortex fields $\langle\omega\rangle$, are obtained and interpreted time averaged velocity vectors $\langle\mathrm{V}\rangle$, averaged streamlines $\langle\Psi\rangle$, and averaged vortex fields $\langle\omega\rangle$, changes with the turbine blade positions. As a result, it is seen that turbine azimuthal angle affetcs the flow structure and streamline focal points, positive and negative vortex are formed in the flow region behind the turbine. Only one focal point at $\theta=240^{\circ}$ is occurred while two focal points and one stagnation point are occurred at the angle of $\theta=270^{\circ}$.
\end{abstract}

Keywords: Darrieus Water Turbine; Energy; Hydraulic Energy; Renewable Energy; PIV

\section{Introduction}

In the current century, the world continues to develop and grow rapidly in terms of industry, technology and population. As a result of these developments, people's need and demand for energy is also increasing. The most important part here is to meet the increasing energy demand without external dependence or by keeping the dependence at a minimum level. Because independence in energy is a symbol of power among the countries of the world. Fossil based fuels, which are still in use from the past to the present, have an important place in energy production, but due to the decrease in fossil based fuel reserves and the damage to the environment and human health, different energy production methods have been investigated and interest in renewable energy has increased in recent years. The most important reasons for the orientation to renewable energy are that it has the potential to produce continuous energy, as well as does not cause any harm to the environment and people. Renewable energy sources can be named and classified as wind, solar, dams, streams, tides and geothermal. It is very important to benefit from these energy resources in the most efficient way. Therefore, various studies are carried out in order to increase energy production efficiency and studies carried out for this purpose are supported by the world countries. Turkey also continues to develop in terms of Industry, population and technology, and accordingly, energy needs and demand are increasing. Turkey's largest fossil fuel reserves to produce energy internal power plant are lignite and hard coal. However, their environmental pollution and harm to human health should be reduced and this increasing demand should be supply by reducing external dependency. For this reason, 
studies and researches in the field of renewable energy have increased. Şekeroğlu, (2019), studied a $5 \mathrm{~kW}$ Darrieus turbine based on the dimensions of a open channel with 2.2$2.5 \mathrm{~m} / \mathrm{s}$ flow velocity in Adana. According to the calculated values, he designed a prototype using a $3 \mathrm{D}$ modeling program. A $1 / 4$ scale model of a prototype for experimental flow analysis of a turbine has been produced and four different azimuth angle, $\theta=270^{\circ}, \theta=240^{\circ}, \theta=210^{\circ}, \theta=180^{\circ}$ and investigated the changes in the flow around the turbine blades in experiments using the PIV method for different Re, numbers [1]. Yanıktepe, et al. (2017), solar energy production and economic evaluation of the daily solar radiation in Turkey and have analyzed the average temperature data measured over three years. In their study, they used a photovoltaic (PV) based grid connected to a power plant with an installed capacity of $1 \mathrm{MW}$ in electricity generation. As a result of their studies and calculations, they showed that Osmaniye is an important region for PV investment in electricity production. In addition, they also predicted the future of the use of fossil fuels and renewable energy sources and systems in energy production [2]. Also Yanıktepe, et al. (2013), using the Weibull and Rayleigh distribution methods, examined the wind energy potential in Osmaniye and gave information about whether the region is suitable for the relevant investments. In this study, between January 2008 and August 2011 by the Turkish state meteorological service for 44 months collected $10 \mathrm{~m}$ high wind speed, direction and flow time consisting of wind data are used [3]. Demircan, (2014), studied Darrieus Rotor type vertical axis water turbine to be used in river flows using Computational Fluid Dynamics (HAD) in his study, he studied the vertical axis water turbine experimentally to verify the two dimensional numerical solution and examined the performance of various vortex models. He designed a vertical axis water turbine using QBlade software and compared the results here with two-dimensional numerical modeling and experimental results [4]. Hwang, et al. (2009), in their study, examined a cycloidal wind turbine mounted on a connected balloon or parafoil as a new high altitude wind power system. In this study, it has been stated that a curtain control mechanism is needed because HAWT's (Horizontal Axis Wind Turbines) generate power in one or two way flow. On the other hand, it has been concluded that VAWT's (Vertical Axis Wind Turbines) can operate independently of the flow direction and without the need for a curtain control mechanism [5]. Alidadi, (2009), investigated channel optimization for vertical axis hydro current turbine with diffuser in his study. In this study, a separate vortex method, called the Discrete Vortex Method (DVM), was developed primarily for the performance analysis of without diffuser vertical axis turbines. By comparing the results of without diffuser and with diffuser turbines, he showed that the diffuser significantly increases the power coefficient of the vertical axis turbine. In addition, the results showed that the diffuser significantly reduced torque fluctuations, especially at high tip speed ratio [6]. Antheaume, et al. (2008), analyzed the efficiency of a cross flow water turbine with the help of simulations for free stream flow conditions against energy farm conditions in these studies. The simulations have shown the advantage of the tower configuration for efficiency. For this reason, the towers were placed in a cluster of several row towers making a barge, and simulations showed that the average barge efficiency increased as the distance between towers decreased and the number of towers increased in line [7]. Li and Calisal, (2010), conducted a study on 3D effects on vertical axis turbines in their study. In the study, the vertical axis tidal current turbine and the newly developed vortex method were used. They specifically analyzed the effects on power output and wake region estimation [8]. Bachant and Wosnik, (2016), carried out their experiments in a large scale laboratory with a vertical axis cross flow turbine with high solidity to establish scale parameters and investigate wake zone properties and Reynolds number on performance. They have shown that the performance of the cross flow turbine becomes essentially Reynolds independent at a Reynolds number, $R e_{C_{L}}=2 \times 10^{5}$ based on an approximate average Reynolds number chord length, or $R e_{D}=10^{6}$ based on rotor diameter [9]. Khan, et al. (2009), based on a comprehensive study of the various hydrokinetic systems reported to date, have deduced general trends in system design, channel magnification, and placement methods. They also provided a detailed assessment of various turbine systems (horizontal and vertical axis) with classification and qualitative comparison [10]. In general, two of the most commonly used renewable energy sources are wind and hydraulic energy. In hydraulic energy, the potential energy of water is allowed to be transformed into kinetic energy, or mechanical energy is obtained from the existing kinetic energy by means of turbines and electricity generation is realized through the alternator. In wind energy, electricity generation is carried out with a similar logic. Classification of wind turbines is made as vertical axis wind turbine (VAWT) and horizontal axis wind turbine (HAWT) depending on the position of their rotors relative to the ground. Vertical axis wind and water turbines (VAWT) and a horizontal axis wind and water turbine (HAWT) are shown in Figure 1.

In addition, Figure 1 shows images of different types of vertical axis turbines. These turbines are (a) Squirrel Cage Darrieus, (b) H-Darrieus, (c) Darrieus, (d) Gorlov and (e) Savonius rotor turbines. In this study, the calculation and design of a three bladed vertical axis darrieus turbine was made that could produce $5 \mathrm{~kW}$ of power from the open channel with a free stream flow rate of 2.2-2.5 m/s. After that, 
two different azimuth angles, $\theta=270^{\circ}, \theta=240^{\circ}$ and changes in flow around the blades of turbine were examined in PIV method experiments for the $\mathrm{Re}=9000$.
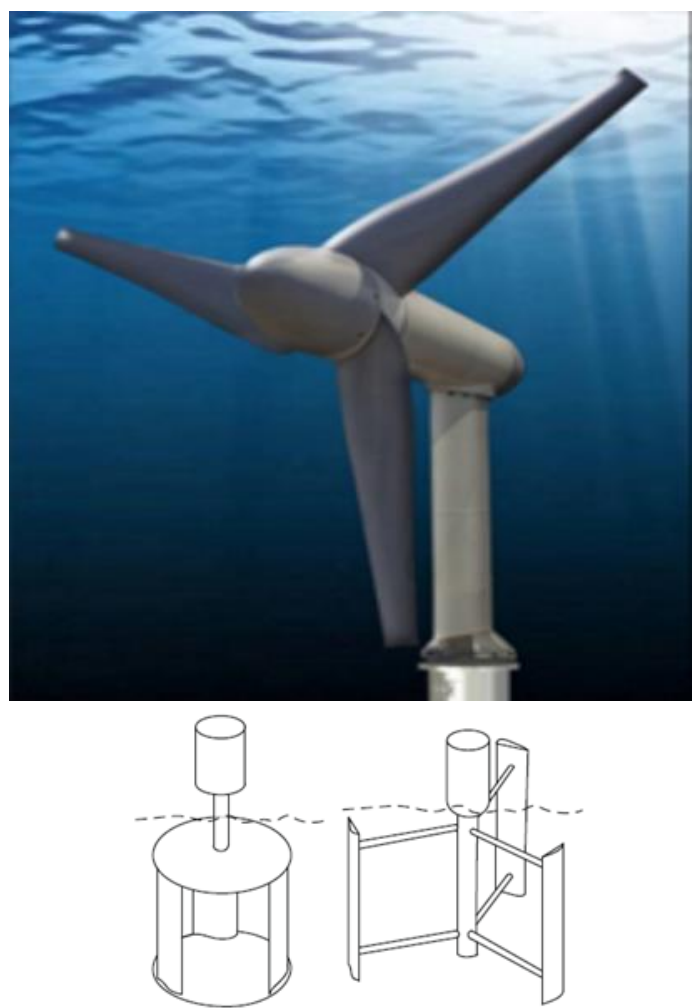

(a) Squirrel Cage Darrieus

(b) H-Darrieus

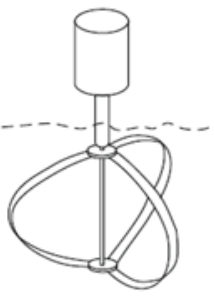

(c) Darrieus

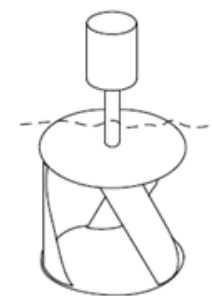

(d) Gorlov

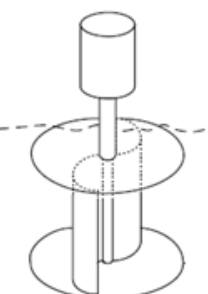

(e) Savonius
Fig. 1. Vertical axis wind and water turbines (VAWT), and a horizontal axis wind and water turbine (HAWT) $[10,11]$

\section{Material and Method}

\subsection{Flow dynamics of the H-Darrieus turbine}

The power that can be obtained by a hydrokinetic turbine from any fluid medium is proportional to the kinetic energy passing through the turbine per unit time and is calculated by the equation given below [6].

$$
P=C_{p} \frac{1}{2} \rho A_{\text {ref }} V_{0}^{3}
$$

$\mathrm{Cp}$ is the power coefficient of the turbine, $\rho$ is the density of the fluid, $\mathrm{V}_{0}$ is free stream flow velocity, $\mathrm{A}_{\text {ref }}$ is turbine sweep area $\mathrm{R}$ is the turbine radius and $\mathrm{H}$ is the turbine blade height. For a vertical axis turbine, $A_{\text {ref }}$ is expressed by the following equation [6].

$$
A_{\text {ref }}=2 R H
$$

Darrieus turbine, which has a simple structure, can be used in wind and water flows. A basic flat blade Darrieus turbine is shown in Figure 2.

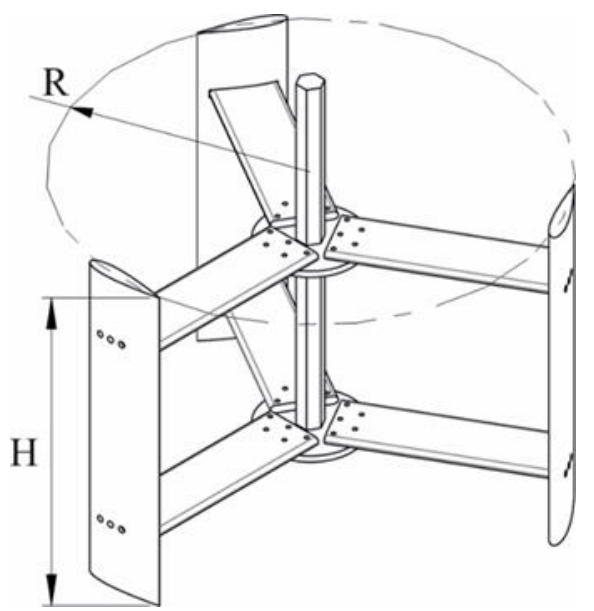

Fig. 2. Darrieus turbine

Although the Darrieus turbine has a simple geometry, the flow area in and around the turbine sweep area is highly unstable and usually three dimensional. Understanding the complex flow structures in the Darrieus turbine is important to increase turbine efficiency. In Figure 3, velocity triangles and azimuth angle positions of Darrieus turbine blade are shown [5].

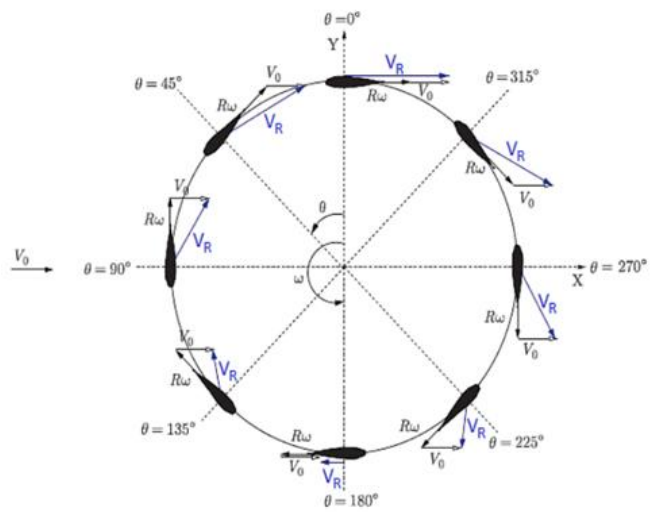

Fig. 3. Azimuthal position and Velocity triangle diagram [5]

As seen in Figure 3, the turbine blade position is defined by the azimuth coordinate $\theta$ [5]. The free stream flow of $V_{0}$ acting on the turbine blades rotates the turbine and as a result, the blades have a tangential linear velocity, expressed by the equation $V_{0}=\omega R$ at the linear velocity. $\omega$ is the angular velocity and $\mathrm{R}$ is the turbine radius. The velocity in an azimuth position for any blade is given below.

$$
V_{R}=\sqrt[2]{\left(V_{0}+V_{\theta} \cos \theta\right)^{2}+\left(V_{\theta} \sin \theta\right)^{2}}
$$

The ratio of tangential velocity to free stream flow velocity gives the tip speed ratio, the tip speed ratio is denoted by $\lambda$ and expressed as,

$$
\lambda=\frac{\omega R}{V_{0}}
$$

The angle of attack is an angular measure of the distance between the $V_{R}$ obtained and the chord line of the blades. Also, the angle of attack is generally the same as the tangential velocity direction for a cross flow turbine. Angle of attack can be calculated with the following equation. 


$$
\alpha=\tan ^{-1}\left(\frac{\sin \theta}{\lambda+\cos \theta}\right)
$$

In another important parameter, the solidity of the turbine is ' $\sigma$ ' and is defined below.

$$
\sigma=\frac{N C_{L}}{R}
$$

$\mathrm{N}$ is the number of blades in the turbine, $\mathrm{C}_{\mathrm{L}}$ is the blade chord length and $\mathrm{R}$ is the radius of the turbine [11].

\subsection{Turbine calculation and design}

In this experimental study, the calculation and design of a three bladed vertical axis darrieus turbine was made that could produce $5 \mathrm{~kW}$ of power from the open channel with a free stream flow rate of $2.2-2.5 \mathrm{~m} / \mathrm{s}$. For this, the necessary numerical calculations were made, respectively, and the dimensions of the $1 / 1$ scale turbine were determined. Later, model production was carried out by reducing it to $1 / 4$ scale for use in experiments. The parameters of the turbine model are given in Table 1.

Table 1. Design parameters of the turbine model

\begin{tabular}{ll}
\cline { 1 - 2 } Design Parameters & \\
\hline Blade Profile & NACA0018 \\
Chord Length, $\mathrm{C}_{\mathrm{L}}$ & $5 \mathrm{~cm}$ \\
Blade Thickness, $\mathrm{t}$ & $0.9 \mathrm{~cm}$ \\
Blade Number, N & 3 \\
Height of Turbine Blad & $20 \mathrm{~cm}$ \\
Turbine Radius, R & $13.75 \mathrm{~cm}$ \\
Rotor Diameter & $3 \mathrm{~cm}$ \\
\hline
\end{tabular}

2.3. Establishing the experimental system and conducting the experiment

\subsubsection{Dye experiment}

The model, which was produced on the water tunnel with free surface flow in the advanced fluid mechanics laboratory at Osmaniye Korkut Ata University, was placed in the appropriate position. The water tunnel has two water tanks, and between these tanks there is an acrylic test zone with dimensions of 6000x800x1000mm (length $\mathrm{x}$ width $\mathrm{x}$ height). The flow rate in the water tunnel is adjusted by two axial pumps that can rotate at different revolutions with the help of frequency control unit. In the experiment, a reference blade was determined in order to determine the blade positions. As the first position, the reference blade is positioned so that the flow is exactly perpendicular. The initial position of the reference blade is at position $270^{\circ}$ according to the azimuthal position and velocity triangle scheme. Images were taken by using rhodamine, a special fluorescent dye, and providing the glow of the dye with the help of laser. The Canon EOS M50 digital camera is used to capture images conducted dye experiments. In this way, the flow structure around the blade and in its wake region was observed, and depending on these data, the imaging area was determined for the Particle Imaging Velocity Measurement (PIV) experiment.

\subsubsection{Particle Imaging Velocimetry (PIV) Experiment}

In order to analyze the flow characteristics and measure the speed using the PIV technique, metal or plastic particles coated with shiny silver with a diameter of 10-20 micrometers, the density of which is almost the same as the density of the water, are added to the cycle water. Although the density of the particles is slightly higher than the density of water, they can move at the same speed (varying in the range of $1000-1100 \mathrm{~kg} / \mathrm{m}^{3}$ ).
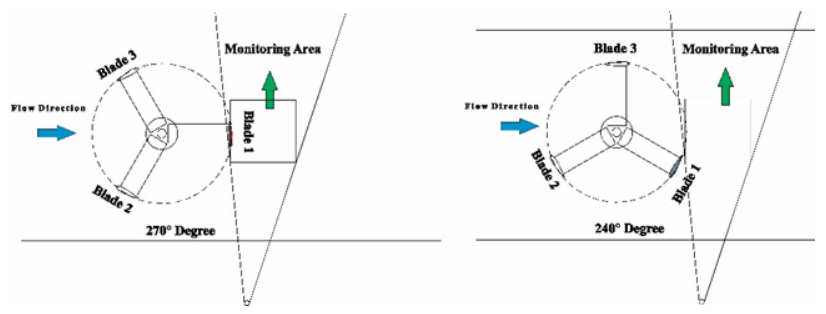

Fig. 4. Monitoring area and Blade positions during the experiment

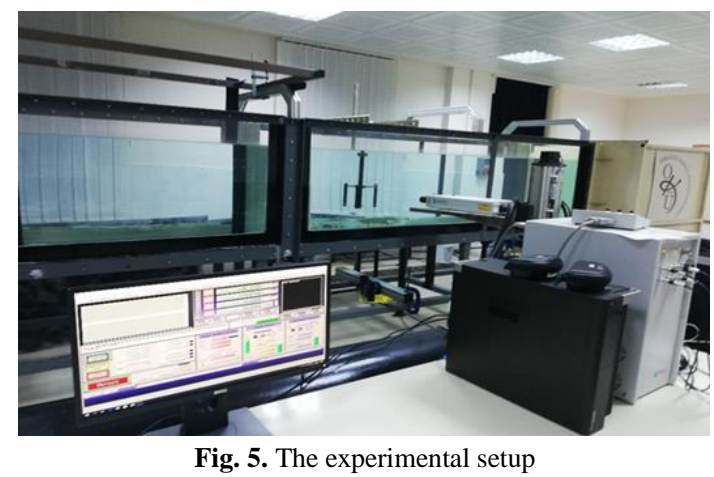

The laser beam produced by the double pulsed $145 \mathrm{~mJ}$ laser source (Nd: YAG Laser) is transformed into a laser beam of 1-2 mm thickness and different widths with the help of optical equipment. The laser source can generate a maximum of 15 laser beam pairs per second. The flow illuminated by laser beams is transferred to the computer environment with two photographs of the particles moving in the flow field with time intervals of milliseconds $(\Delta t)$ taken with the help of a digital camera. The time $(\Delta \mathrm{t})$ between two photos depends entirely on the velocity of the flow and the level of turbulence. Because the model of the laser source is pulsed, the laser and camera must work in combination. The device that provides the combination between the laser source and the digital camera is called a synchronizer and is controlled by a computer. These photos, which are transferred to the computer environment, are mostly divided into small subregions of $32 \times 32$ pixel dimensions (these dimensions can be reduced or increased when necessary) and the $\Delta t$ time interval between both photos and the average distance traveled by the particles in each sub-region is known, so the velocity of the flow in the sub-regions considered is calculated. In the reference flow region, $\mathrm{m} * \mathrm{n}$ velocity vectors are calculated according to the size of the photo area. The turbine is positioned in the water channel at the desired position and perpendicular to the flow in order to perform the flow analysis of the turbine with the PIV method in the closed 
system open water channel. Turbine positions in static states are $270^{\circ}$ and $240^{\circ}$ according to the position of the reference blade. The schematic view of the flow direction, blade positions and monitoring areas is given in Figure 4. The image of the experimental setup is given in Figure 5.

\section{Results and Discussions}

In this study, the results of dye and PIV experiments of a vertical axis water turbine are given. The dye experiment was single and the PIV experiment was also performed at two different azimuth angles and at the value of $\mathrm{Re}=9000$. The turbine blades are given an attack angle of $\alpha=-2^{\circ}$. In figure 6 , dye experiments performed for visualization of the flow around the blade are given and the time gap between photos was determined as $\Delta \mathrm{t}=0.047$ seconds

When the images of the dye experiment at $270^{\circ}$ position of the blade referenced in Figure 6 are examined, the formation of vortices is clearly observed in the wake region behind the turbine blade, and the vortices are lost by moving in the direction of flow. It has been observed that the vortices formed clockwise and counterclockwise. At $C_{L} / L=1$, flow stagnation is observed (L; Distance flow direction from turbine blade center) in the blade wake region. Figure 7 and Figure 8, the results of the PIV experiments are given. The order of data in the visuals of the PIV experiment is averaged velocity vectors $\langle\mathrm{V}\rangle$, averaged streamlines $\langle\psi\rangle$ and averaged vortex fields $<\omega>$.
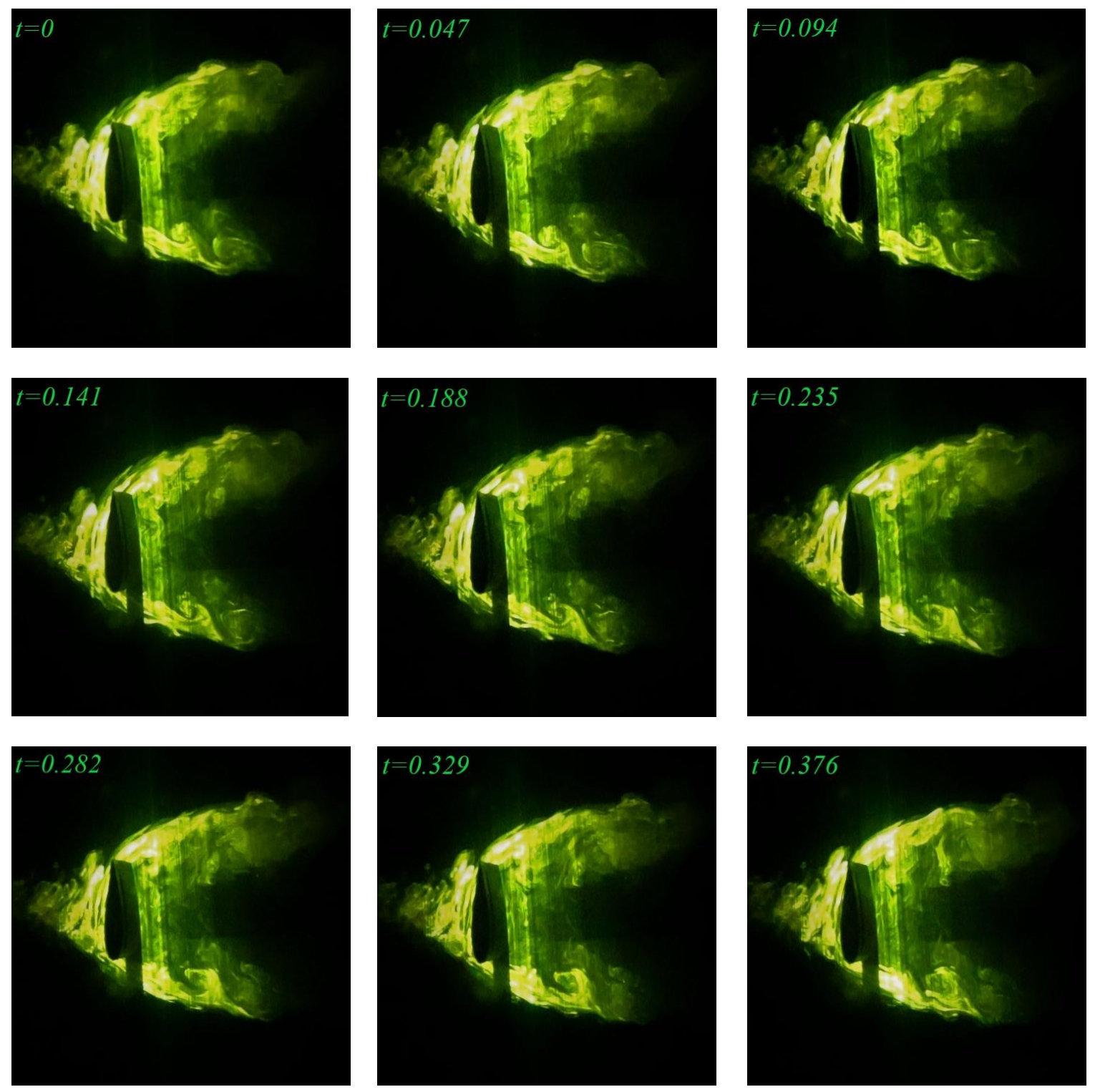

Fig. 6. Dye images at $\operatorname{Re}=9000$ and $\theta=270^{\circ}$ 

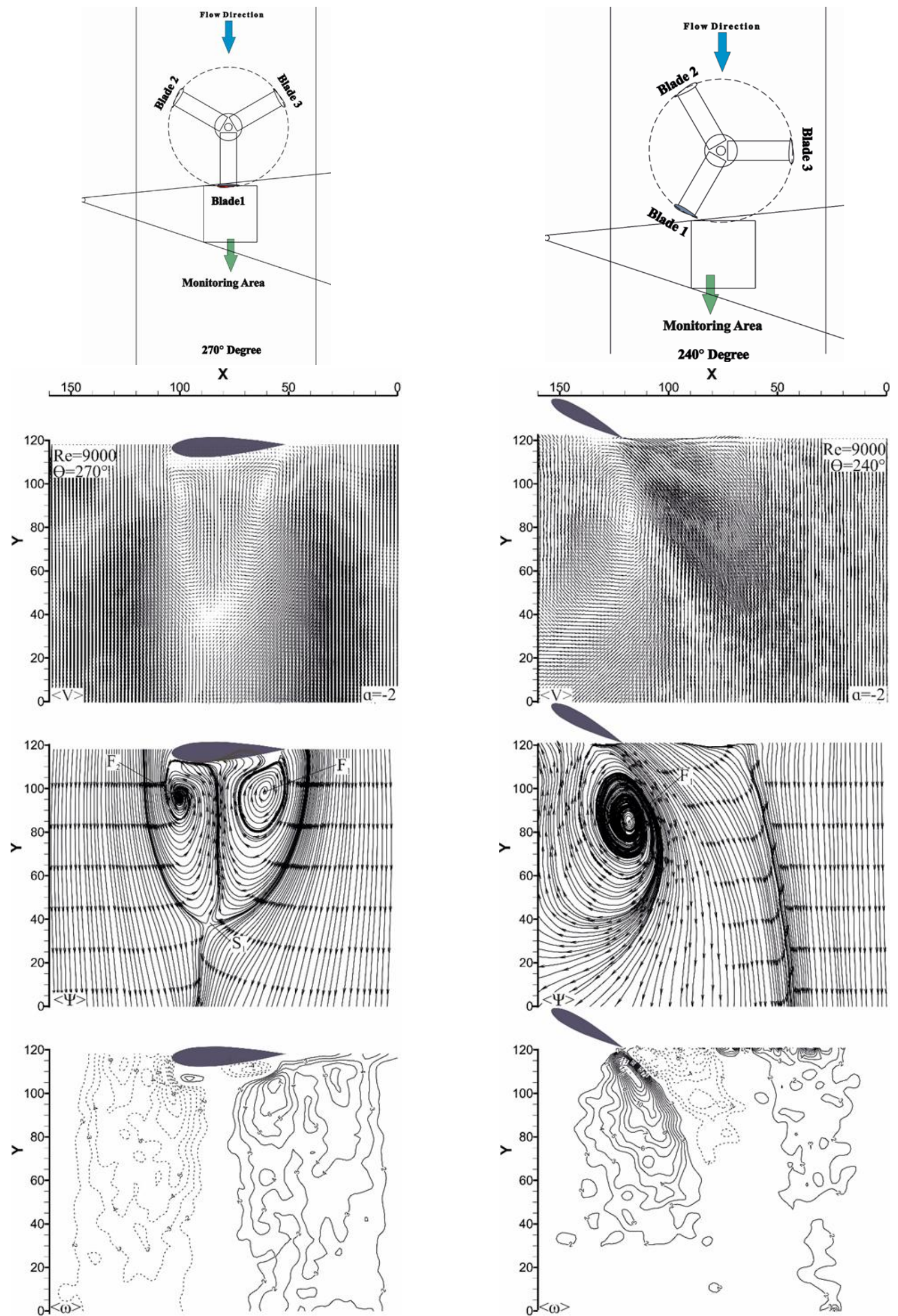

Fig. 7. At $\mathrm{Re}=9000$ and $\theta=270^{\circ}$ avarage velocity vectors $<\mathrm{V}>$, streamlines $<\psi>$ and vortex contours $\langle\omega\rangle$

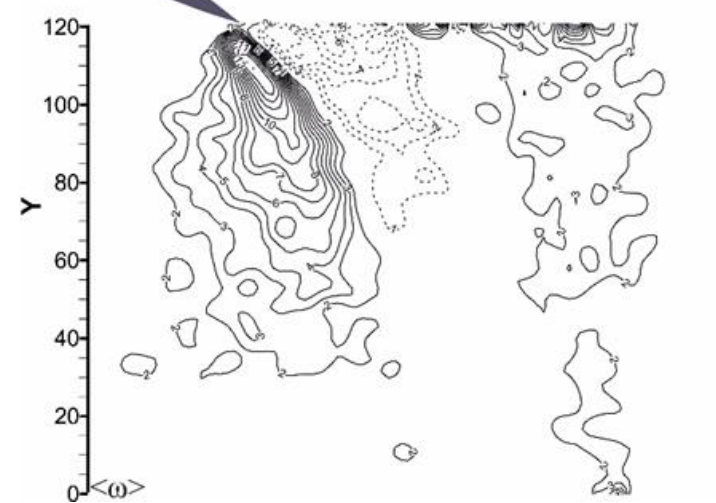

Fig. 8. At $\mathrm{Re}=9000$ and $\theta=240^{\circ}$ avarage velocity vectors $<\mathrm{V}>$, streamlines $\langle\psi\rangle$ and vortex contours $\langle\omega\rangle$ 
When the time-averaged velocity vectors $\langle\mathrm{V}\rangle$, are examined in Figure 7, it is observed that the velocity values in the wake regions of the turbine blade are low, the velocity values approach the free stream flow velocity as they move away from the turbine blade. $\mathrm{V}_{\max }=44.0461 \mathrm{~mm} / \mathrm{s}$ and $\mathrm{V}_{\min }=-$ $211.594 \mathrm{~mm} / \mathrm{s}$ were observed in the flow area. Just behind the blade, the formation of a vortex flow region is observed in the area of $x=100 \mathrm{~mm}, y=110 \mathrm{~mm}$ and $x=50 \mathrm{~mm}, y=40$ $\mathrm{mm}$. When the average streamlines $\langle\psi\rangle$, are examined, it is observed that two distinct streamline focal points (Foci, $F_{1}$ and $F_{2}$ ) occur and a stagnation point $\left(S_{1}\right)$ is formed. $F_{1}$ is formed at the point $\mathrm{x}=61.1004 \mathrm{~mm}, \mathrm{y}=98.9385 \mathrm{~mm}$ and $\mathrm{F}_{2}$ at the point $\mathrm{x}=100.23 \mathrm{~mm}, \mathrm{y}=96.3298 \mathrm{~mm}$. In addition to these, $\mathrm{S}_{1}$ occurs at the point $\mathrm{x}=86.1837 \mathrm{~mm}, \mathrm{y}=37.936 \mathrm{~mm}$. When the average vortex fields $\langle\omega\rangle$, is examined, it is seen that negative and positive vortex regions are formed. Vortex contours vary between minimum -10 and maximum +10 ranges and the amount of increase is 1 . It is seen that the vortex start just behind the blade and progress symmetrically to the end of the field of view. It is observed that the vortex region moving down from the attack edge of the blade is negative, and a positive vortex region is formed on the trailing edge.

When the time-averaged velocity vectors $\langle\mathrm{V}\rangle$, are examined in Figure 8, it is seen that the velocity values in the turbine blade trailing area are higher than the free stream flow velocity, while the velocity values just behind the turbine blade approach the free stream flow velocity. Just behind the blade, the formation of a vortex flow region is observed in the area of $x=115 \mathrm{~mm}, y=115 \mathrm{~mm}$ and $x=160 \mathrm{~mm}, y=0 \mathrm{~mm}$. $\mathrm{V}_{\max }=64.4582 \mathrm{~mm} / \mathrm{s}$ and $\mathrm{V}_{\min }=-189.933 \mathrm{~mm} / \mathrm{s}$ were observed in the flow area. When the average streamlines $\langle\psi\rangle$ are examined, it is observed that one $F_{1}$ is formed. $F_{1}$ is formed at the point $\mathrm{x}=118,188 \mathrm{~mm}, \mathrm{y}=85,333 \mathrm{~mm}$. When the average vortex fields $\langle\omega\rangle$, is examined, it is seen that negative and positive vortex regions are formed. Vortex contours vary between minimum -7 and maximum +15 ranges and the amount of increase is 1 . Just behind the trailing edge of the blade, the positive and negative vortex region symmetrically separated by the trailing edge are clearly visible. Except for small fragments, it has a single piece and a smooth shape. The positive vortex region on the right side of the monitoring area is also created by the effect of the turbine rotor.

\section{Conclusions}

Experiments were carried out using the PIV method for Re, 9000 number and two different azimuth angles, $\theta=270^{\circ}$, $\theta=240$. Then the changes in the flow around the turbine and its blades were investigated. These are given as timeaveraged velocity vectors $\langle\mathrm{V}\rangle$, average streamlines $\langle\psi\rangle$ and average vortex fields $\langle\omega\rangle$. Result show that high velocity vector changes with the angle of turbine blades. it is observed that the velocity values in the wake regions of the turbine blade are low, the velocity values approach the free stream flow velocity as they move away from the turbine blade. When turbine azimuth angle is $\theta=270^{\circ}$, the flow velocity in wake regions of the blade is getting descrease. It was observed that the focal points of these vortices, $F_{1}$ and $F_{2}$, were symmetrically formed, where negative and positive vortices were formed in the blade wake region at the position $\theta=270^{\circ}$ in the average streamlines $\langle\psi\rangle . F_{1}$ is formed at the point $\mathrm{x}=61.1004 \mathrm{~mm}, \mathrm{y}=98.9385 \mathrm{~mm}$ and the other focal point, $F_{2}$, which is symmetrical to the $F 1$ is observed at the point $\mathrm{x}=100.23 \mathrm{~mm}, \mathrm{y}=96.3298 \mathrm{~mm}$. In addition, stagnation point $(S)$ occured at the point of $x=86.1837 \mathrm{~mm}, y=37.936$ $\mathrm{mm}$. One focal point $\left(\mathrm{F}_{1}\right)$ is visible at $\theta=240^{\circ}$ position While two focal point (F1 and F2) and one stagnation point are observed at $\theta=270^{\circ}$. When the average vortex fields $\langle\omega\rangle$ are examined, it is seen that negative and positive vortex regions are formed and the positive vortex area on the right side of the monitoring area is created by the effect of the turbine rotor. Focal point, $F_{1}$, at $\theta=240^{\circ}$ is formed at the point $\mathrm{x}=118,188 \mathrm{~mm}, \mathrm{y}=85,333 \mathrm{~mm}$.

\section{Acknowledgment}

This study was supported by OKU Scientific Research Projects Unit with the project number OKU BAP-2019-PT3015 and supported by TÜBITAK TEYDEB with project number 7170525, we thank both institutions for their support. In addition, we would like to thank the project owner ECC Machine Chemistry Energy Industry and Trade Limited Company for their support.

\section{ORCID}

Bülent Yanıktepe

Ertuğrul Şekeroğlu

Mustafa Söyler

Coşkun Özalp

(iD) $0000-0001-8958-4687$
(iD) $0000-0002-6592-3872$
(iD) $0000-0003-4767-5825$
(iD) $0000-0003-2249-7268$

\section{References}

[1] Şekeroğlu, E. 2019. "Investigation of flow and power parameters of a vertical Axis water turbine for stream application". Master thesis, Osmaniye Korkut Ata University, Energy System Engineering Department, Osmaniye, Turkey, 1-85.

[2] Yaniktepe, B., Kara, O. and Ozalp, C., 2017. Technoeconomic evaluation for an installed small-scale photovoltaic power plant. International Journal of Photoenergy, 1-7.

[3] Yaniktepe, B., Koroglu, T. and Savrun, M. M. 2013. Investigation of wind characteristics and wind energy potential in Osmaniye, Turkey. Renewable and Sustainable Energy Reviews, 21, 703-711. 
[4] Demircan, E. 2014. "Vertical axis water turbine design and analysis for river applications using computational fluid dynamics". Master Thesis, METU Mechanical Engineering Department, Ankara, Turkey, 57.

[5] Hwang, I. S., Lee, Y. H., Kim, S. J., Optimization of cycloidal water turbine and the performance improvement by individual blade control. Applied Energy, 86(9), 1532-1540, 2009.

[6] Alidadi, M. 2009. "Duct optimization for a ducted vertical axis hydro current türbine". Ph.D. Thesis, The University of British Columbia, Vancouver, 1-122.

[7] Antheaume, S., Maître, T. and Achard, J. L. 2008. Hydraulic Darrieus turbines efficiency for free fluid flow conditions versus power farms conditions. Renewable Energy, 33(10), 2186-98.

[8] Li, Y. and Calisal, S. M. 2010. Three dimensional effects and arm effects on modeling a vertical axis tidal current turbine. Renewable Energy, 35(10), 2325-2334.

[9] Bachant, P. and Wosnik, M. 2016. Effects of reynolds number on the energy conversion and near wake dynamics of a high solidity vertical axis cross flow türbine. Energies, 9(2), 1-18.

[10] Khan, M. J., Bhuyan, G., Iqbal, M. T. and Quaicoe, J. E. 2009. Hydrokinetic energy conversion systems and assessment of horizontal and vertical axis turbines for river and tidal applications. A technology status review. Applied Energy, 86(10), 1823-1835.

[11] Hall, T.J. 2012. "Numerical simulation of a cross flow Marine Hydrokinetic turbine", Master Thesis, Mechanical Engineering University of Washington, Washington, 1-95. 